\title{
Consumo de oxígeno en Perumytilus purpuratus según el parasitismo por Digenea, $y$ efectos de la temperatura y del ciclo de emersión-inmersión sobre la liberación de cercarias
}

Oxygen consumption in the mussel Perumytilus purpuratus according to the parasitism by Digenea, and effects of temperature and the emersion-submersion cycles on the shedding of cercariae

\section{Mauricio Castro-Rojas ${ }^{1}$, Katherine Cid-Moya ${ }^{1}$ y Mario George-Nascimento ${ }^{1}$}

'Departamento de Ecología, Facultad de Ciencias, Universidad Católica de la Santísima Concepción, Casilla 297, Concepción, Chile.mcastrorojas@ucsc.cl

\begin{abstract}
Digenea are common platyhelminth parasites in intertidal organisms; their free-living stages (miracidia and cercaria) are not free of experiencing the effects of physico-chemical variations of this environment, same as their hosts. Moreover, the hosts may show different physiological behavior depending on if they are or not parasited. This study deals with the effects that a yet unidentified digenean may have upon the oxygen consumption of Perumytilus purpuratus, as well as the effects that may have the emersion-immersion cycles in the laboratory, on cercariae release. To do this, individuals of P. purpuratus were collected by hand from intertidal rocky platforms nearby the mouth of the Biobío River, Chile. In the laboratory, oxygen consumption was compared between hosts with and without parasites, and the shedding of cercariae was measured at 9,17 y $22^{\circ} \mathrm{C}$ and compared between parasitized hosts exposed to opposite emersion-submersion cycles. There were no significant differences between parasitized and non-parasitized hosts in oxygen consumption. However, parasitized hosts showed scarce gonadic development, suggesting that metabolic products of the hosts are re-allocated towards parasite development. Cercariae shedding increased with water temperature and decreased exponentially with the duration of the submersion- emmersion cycle, peaking $2 \mathrm{~h}$ after submersion. These results suggest that metabolic rate of the host increases with temperature and that cercariae release is induced by temperature and submersion of the host in seawater.
\end{abstract}

Key words: Fellodistomidae, host physiology, parasite ecophysiology, intertidal, Chile

Resumen.- Los Digenea son platelmintos parásitos comunes en organismos intermareales, y en sus etapas de vida libre (miracidio y cercaria) están expuestos a distintas condiciones ambientales, al igual que sus hospedadores. Por otro lado, éstos últimos pueden presentar un comportamiento fisiológico diferente si están o no parasitados. En este estudio se evaluó el efecto de la parasitosis por un digeneo, aún no identificado, sobre el consumo de oxígeno del chorito Perumytilus purpuratus, y se analizó la influencia de la temperatura del agua y los ciclos de emersión-inmersión sobre la liberación de las cercarias de estos parásitos. Ejemplares de P. purpuratus fueron recolectaron manualmente desde el roquerío intermareal de la desembocadura del río Biobío, Chile. En el laboratorio se comparó, en experimentos con distintos ejemplares, el consumo de oxígeno de individuos con y sin parásitos, y se midió la liberación de cercarias a 9,17 y $22^{\circ} \mathrm{C}$ de temperatura del agua, y según ciclos opuestos de inmersión-emersión de duración variable. El consumo de oxígeno no mostró diferencias significativas entre choritos parasitados y no parasitados. Sin embargo, los ejemplares parasitados mostraron escaso desarrollo gonadal, lo que sugiere una reasignación de los productos metabólicos de los choritos hacia el parásito. La liberación de cercarias aumentó con la temperatura del agua y decreció exponencialmente con la duración de la inmersión-emersión, alcanzando valores máximos en las primeras $2 \mathrm{~h}$ de ocurrida la inmersión. Las secuencias opuestas de los ciclos de inmersión-emersión empleadas en los experimentos no influyeron significativamente en el número final de cercarias liberadas. Estos resultados sugieren que el metabolismo del hospedador aumenta con la temperatura, y que la inmersión y la temperatura del agua inducen la liberación de las cercarias.

Palabras clave: Fellodistomidae, fisiología del hospedador, ecofisiología de parásitos, intermareal, Chile 


\section{INTRODUCCIÓN}

El parasitismo por Digenea (Platyhelminthes: Trematoda) es un fenómeno ampliamente extendido en moluscos, ya que éstos son generalmente su primer hospedador intermediario, y en algunos casos incluso pueden ser su segundo hospedador intermediario. En los moluscos ocurre la reproducción asexual del parásito, particularmente en la glándula digestiva y en las gónadas del hospedador, la que usualmente resulta en la producción y liberación de larvas cercarias al ambiente (Noble \& Noble 1965). El parasitismo de digeneos en moluscos, generalmente altera el desarrollo, función de defensa, nutrición, metabolismo y reproducción del hospedador (Cheng 1967). De hecho, un resultado común de estas parasitosis es la castración total o parcial del hospedador. Por lo expuesto, pueden influir en varios aspectos de la historia de vida de sus hospedadores y determinar su distribución y abundancia (Fredensborg et al. 2005).

En general, las asociaciones de los digeneos con moluscos gasterópodos han sido las más estudiadas porque son las más extendidas y primitivas (Cribb et al. 2001), por lo que hay menos estudios parasitológicos en los moluscos bivalvos. El creciente interés por este tipo de sistemas de interacciones interespecíficas se ha centrado en el escenario del cambio climático global (Marcogliese 2001, Poulin 2006, Marcogliese 2008, Studer et al. 2010). Los parásitos con ciclos de vida complejos pueden verse particularmente afectados por los cambios climáticos, en especial por el efecto que tiene la temperatura sobre ellos mismos y sus hospedadores (Shim et al. 2013). En digeneos se postula que lo anterior está asociado a un incremento en la transmisión entre sus hospedadores, ya que la mayoría de los estudios indican un aumento en la liberación de cercarias desde sus hospedadores moluscos (Poulin 2006). Sin embargo, son diversos los factores ambientales que pueden influir sobre la producción y liberación de las cercarias, e.g., pH, incremento de luz y de salinidad, ciclo de mareas (Marcogliese 2001, Mouritsen 2002, Fingerut et al. 2003, Koprivnikar \& Poulin 2009a). Los registros más frecuentes se refieren al aumento de la producción de cercarias con el aumento de la temperatura, aunque hay considerable variación entre las especies de Digenea, y entre los individuos hospedadores (Meyrowitsch et al. 1991, Lo \& Lee 1996, Lyholt \& Buschmann 1996, Abdul-Salam et al. 1997, Cremonte et al. 2001, Fried et al. 2002, Fredensborg et al. 2005, Koprivnikar \& Poulin 2009a, b; Morley 2011, Shim et al. 2013). El único estudio en Chile del efecto del parasitismo por Digenea sobre el metabolismo de sus hospedadores mostró que los hospedadores parasitados tenían un mayor consumo de oxígeno que los no parasitados (Olmos \& GeorgeNascimento 1997).

En esta investigación se evaluó si el parasitismo por larvas de digeneos afecta el consumo de oxígeno del chorito maico Perumytilus purpuratus (Lamarck, 1819) (Bivalvia: Mytilidae), y si la temperatura del agua y la secuencia y duración de los ciclos de emersión-inmersión afectan la liberación de cercarias. El chorito maico es una de las especies más comunes del intermareal rocoso medio de la costa de Chile (Broitman et al. 2001), y es hospedador de al menos 3 taxa de Digenea. Estos son Prosorhynchoides carvajali Muñoz \& Bott, 2011 (Bucephalidae), Proctoeces sp. Odhner, 1911 (Fellodistomidae), y otro digeneo fellodistómido no identificado (Muñoz et al. 2013a), y al que diversos autores han denominado Proctoeces lintoni Siddiqi \& Cable 1960 (Aldana et al. 2009, Oliva et al. 2010), aunque aún no ha sido posible determinar taxonómicamente su identidad en forma definitiva (Muñoz et al. 2013a, b). Este último taxón es el de mayor prevalencia en $P$. purpuratus en la costa centro sur de Chile, alcanzando valores menores al 2\% (Muñoz et al. 2013b), y es el parásito sujeto de este estudio.

\section{MATERIALES Y MÉTODOS}

Entre marzo y mayo 2011 se recolectaron manualmente, durante marea baja, varios miles de ejemplares de Perumytilus purpuratus desde el roquerío intermareal ubicado al norte de la desembocadura del río Biobío (3648’22”'S - 73¹0’38”O), en la región del mismo nombre, Chile. Los individuos fueron llevados al laboratorio en contenedores de plástico de $20 \mathrm{~L}$ con agua de mar, para su posterior revisión de parásitos. Luego, fueron mantenidos individualmente en recipientes de $150 \mathrm{~mL}$ con agua de mar a temperatura ambiente, en un rango entre $\operatorname{los} 14^{\circ}$ a $17^{\circ} \mathrm{C}$ y con aireación constante, emulando ciclos de marea artificiales por períodos de $12 \mathrm{~h}$ sumergidos, 12 $\mathrm{h}$ emergidos. Los recipientes en que los choritos fueron mantenidos individualmente fueron dispuestos en grupos de 50 a 100 individuos por bandeja. La alimentación de los choritos se hizo con microalgas de Dunaliella salina cultivadas en laboratorio, en un medio salino enriquecido, con aireación constante y temperatura ambiente ( $c a$, $\left.15^{\circ} \mathrm{C}\right)$. 
La detección de choritos parasitados se realizó mediante la inspección a ojo desnudo del fondo del envase en que fue puesto cada espécimen, ya que de los hospedadores parasitados emergen cercarias con colas esféricas de color anaranjado, fácilmente visibles por su gran tamaño (Muñoz et al. 2013a). Los individuos no parasitados fueron llevados a su lugar de origen, a excepción de los que se emplearon en los experimentos de consumo de oxígeno.

La comparación del consumo de oxígeno de $P$. purpuratus se efectuó entre 12 individuos parasitados y 15 no parasitados, de similar longitud valvar máxima (entre 15 y $30 \mathrm{~mm}$ ), y su medición se realizó en un equipo FIBOX ${ }^{\circledR}$ 3 de un canal de fibra óptica de medidor de oxígeno en cámaras de $145 \mathrm{~mL}$ de capacidad. La calibración del equipo se realizó entre muestras para cada medición; la primera calibración al $0 \%$ de oxígeno se realizó con saturación de $\mathrm{NO}_{2}$ y la segunda, al $100 \%$ de oxígeno, con agua de mar filtrada con aireación constante a una temperatura de $15^{\circ} \mathrm{C}$. Previo a las mediciones de consumo de oxígeno, los individuos fueron limpiados (extracción de epífitos), aclimatados por 1 semana en cámara fría a $15^{\circ} \mathrm{C}$, con agua de mar filtrada, aireación constante y sin alimentación. Antes de las mediciones de consumo de $\mathrm{O}_{2}$ se obtuvo el peso húmedo de los individuos en una balanza analítica. Luego de las mediciones de consumo de $\mathrm{O}_{2}$, se verificó que los choritos parasitados y no parasitados estaban correctamente catalogados a través de la inspección de los ejemplares bajo lupa. Luego, los individuos fueron secados a $80^{\circ} \mathrm{C}$ durante $48 \mathrm{~h}$ en una estufa de secado (Memmert UF - single DISPLAY), y su peso seco fue medido en una balanza analítica con una sensibilidad de $0,001 \mathrm{~g}$.

Para evaluar el efecto de la temperatura sobre la producción de cercarias, se contaron diariamente el número de cercarias liberadas en 13 choritos a temperatura ambiente $\left(17^{\circ} \mathrm{C}\right)$, durante 5 días. Los organismos fueron sumergidos durante la noche y el conteo se realizó en la mañana del día siguiente. Los datos de liberación de cercarias fueron expresados como número de cercarias por chorito por día. Posteriormente, los mismos individuos fueron sometidos a temperatura de $22^{\circ} \mathrm{C}$ que fue alcanzada paulatinamente $\left(1^{\circ} \mathrm{C} * \mathrm{~h}^{-1}\right.$, durante $\left.5 \mathrm{~h}\right)$ para minimizar el estrés, y luego mantenida constante durante 5 días. Antes de someter a los individuos a $9^{\circ} \mathrm{C}$, se les mantuvo por 36 $\mathrm{h}$ a temperatura ambiente utilizando el mismo procedimiento de bajar en un grado por intervalos de $1 \mathrm{~h}$ la temperatura del agua.
Para discernir si la liberación de cercarias está asociada al acto de inmersión o al tiempo en que el hospedador permanece sumergido, se midió el efecto de la extensión y de la secuencia de los ciclos de emersión-inmersión en 14 ejemplares parasitados que fueron expuestos a ciclos artificiales de 'marea' de distinta duración y dirección. Para ello, se usaron 2 secuencias opuestas de ciclos de inmersión-emersión en los mismos individuos. Los intervalos de tiempo que los organismos estuvieron emergidos-sumergidos fueron de 48, 24, 12, 6, 4, 2 y $1 \mathrm{~h}$. Por ejemplo, en el primer experimento de la serie, los individuos estuvieron $48 \mathrm{~h}$ emergidos (Em) y $48 \mathrm{~h}$ sumergidos $(\mathrm{Su})$. Luego, así también para cada una de los demás experimentos $(24,12,6,4,2$ y 1$)$. Este experimento se llevó a cabo en 2 secuencias, una ascendente, que se refiere a que los ciclos de marea comenzaron desde $1 \mathrm{~h}$ (Em) y $1 \mathrm{~h}(\mathrm{Su})$ hasta completar la secuencia completa y una secuencia descendente, que comenzó con 48 h (Em) - $48 \mathrm{~h}(\mathrm{Su})$ hasta llegar a $1 \mathrm{~h}(\mathrm{Em})$ - $1 \mathrm{~h}(\mathrm{Su})$. Cada secuencia, ascendente y descendente, contempló 194 h de experimentación. La liberación de cercarias fue expresada como número de cercarias liberadas por individuo por hora $\left(\mathrm{N}^{*} \mathrm{~h}^{-1}\right)$.

En el análisis estadístico de los datos se utilizó un análisis de la covarianza del consumo de oxígeno en función del peso seco del mitílido, según la condición de estar o no parasitado. En el caso del efecto de la temperatura sobre la liberación de cercarias, los datos fueron sometidos a un análisis de la varianza de medidas repetidas. Se aplicó la prueba HSD de Tukey para identificar cuáles tratamientos mostraban diferencias significativas (Zar 1999). Finalmente, se utilizó un análisis de la covarianza para evaluar la significancia estadística de las diferencias en la tasa de liberación de cercarias según el tiempo que los hospedadores estuvieron sometidos a 2 regímenes opuestos de emersióninmersión. Todas las pruebas fueron realizadas con los datos transformados al logaritmo decimal.

\section{Resultados}

Las pendientes de las regresiones de la tasa de consumo de oxígeno $\left(\mu \mathrm{mol} \mathrm{O}_{2} \mathrm{~min}^{-1}\right)$ según el peso seco $(\mathrm{g})$ no eran distintas de cero en los ejemplares con y sin parásitos $\left(\mathrm{F}_{1,23}=1,28, P=0,27\right)$. Además, no se encontraron diferencias entre los interceptos de dichas regresiones $\left(\mathrm{F}_{1,24}=0,001, P=0,99\right.$, Fig. 1). Por esto, la ecuación de la regresión lineal $\left.\left(F_{1,25}=0,12\right), R^{2}=0,005\right)$ que describe esta relación para ambos grupos en conjunto (con los errores estándar de los parámetros entre paréntesis) es: 
$\log _{10}$ consumo de oxígeno $\left(\mu \mathrm{mol} \mathrm{O} \min ^{-1}\right)=5,086(0,213)$ $0,069(0,199) * \log _{10}$ peso seco $(\mathrm{g})$.

El análisis de la varianza de medidas repetidas de la liberación de cercarias según la temperatura del agua evidenció variaciones significativas asociadas a ésta $\left(\mathrm{F}_{2}\right.$, $\left.{ }_{36}=22,65, P<0,001\right)$, pero no al efecto intrasujetos $\left(\mathrm{F}_{4,144}=\right.$ $1,56, P=0,19)$. La prueba de Tukey (HSD) mostró que todos los niveles del factor se diferenciaban significativamente $(P<0,05)$, y que liberación de cercarias era mayor a los $22^{\circ} \mathrm{C}$ y menor a $\operatorname{los} 9^{\circ} \mathrm{C}$ (Fig. 2).
Las regresiones de la relación entre la tasa de liberación de cercarias según la duración de la inmersión poseían pendientes negativas, pero que no eran significativamente distintas entre los ejemplares sometidos a secuencias opuestas de ciclos de emersión- inmersión $\left(\mathrm{F}_{1,10}=2,05, P\right.$ $=0,183)$, así como tampoco sus interceptos $\left(\mathrm{F}_{1,11}=0,04, P\right.$ $=0,838)$. En consecuencia, la ecuación que describe dicha relación con ambos experimentos en conjunto (con los errores estándar de los parámetros entre paréntesis) es: $\log _{10} \mathrm{~N} * \mathrm{~h}^{-1}=1,552(0,116)-0,960(0,116) * \log _{10}$ duración del ciclo (h) $\left(R^{2}=0,851\right.$, Fig. 3$)$.

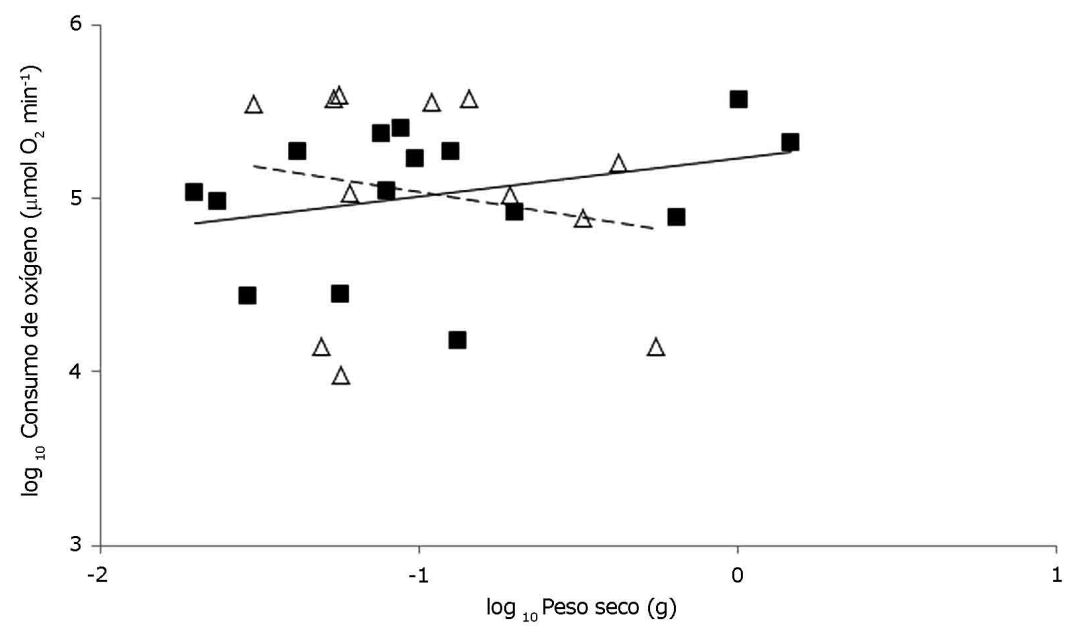

Figura 1. Relación entre el $\log _{10}$ del peso seco $(g)$ de P. purpuratus y el $\log _{10}$ del consumo de oxígeno $\left(\mu \mathrm{mol} \mathrm{O}_{2} \mathrm{~min}^{-1}\right)$ en individuos parasitados (triángulos blancos) y no parasitados con Digenea (cuadrados negros). La línea continua son los no parasitados, y la línea discontinua son los parasitados / Relationship between $\log _{10}$ of the dry weight $(\mathrm{g})$ of P. purpuratus and $\log _{10}$ total oxygen consumption $\left(\mu \mathrm{mol} \mathrm{O}_{2} \mathrm{~min}^{-1}\right)$ in parasitized (white triangles) and non-parasitized with Digenea (black squares). The solid line is for non-parasitized, while the broken line is for parasitized mussels

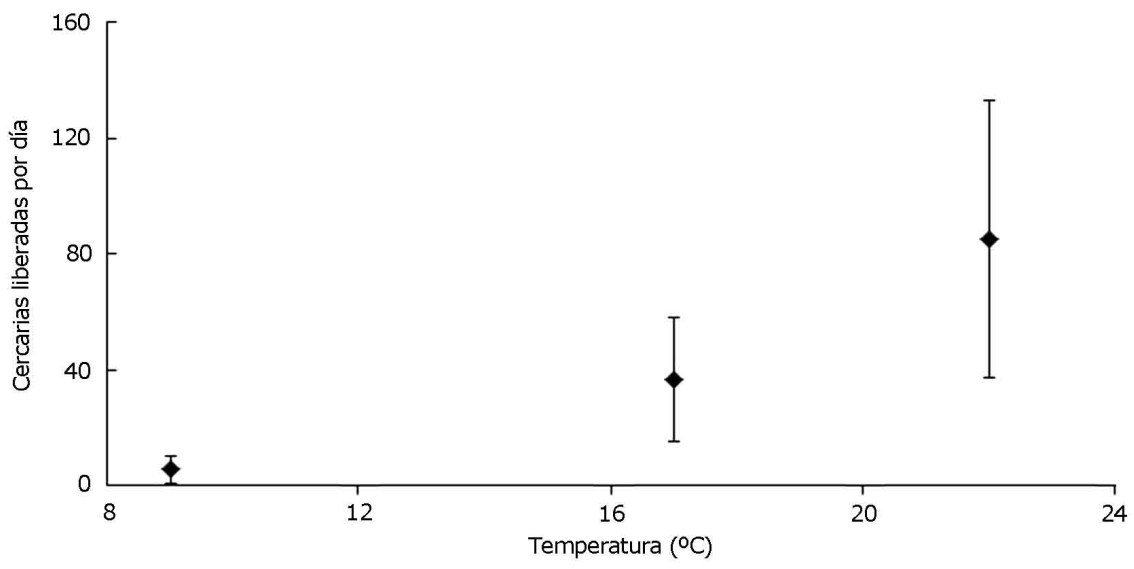

Figura 2. Promedio ( \pm 2 errores estándar) de la liberación diaria de cercarias en 13 ejemplares de Perumytilus purpuratus sometidos a 3 niveles de temperatura por 5 días consecutivos / Mean ( \pm 2 standard errors) of daily emergence of cercariae in 13 Perumytilus purpuratus specimens measured for 3 temperature levels during 5 consecutive days 


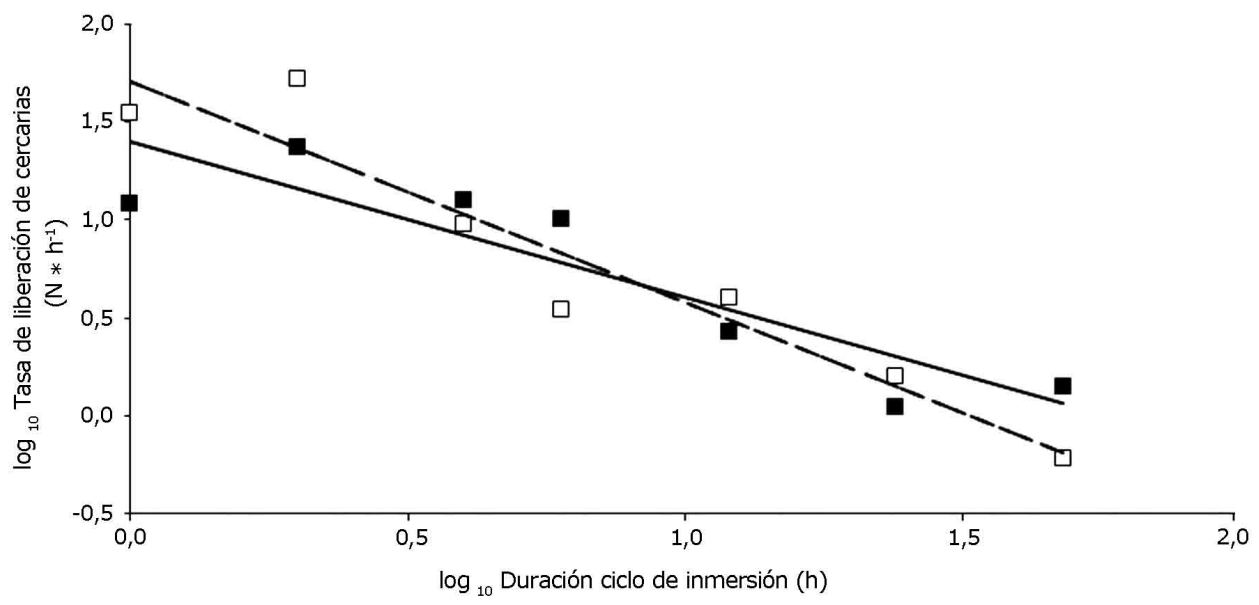

Figura 3. Liberación promedio de cercarias en 14 ejemplares de P. purpuratus sometidos a 2 experimentos con ciclos opuestos de inmersiónemersión. Los cuadrados negros son individuos sometidos a ciclos de inmersión-emersión descendentes en el tiempo (48, 24, 12, 6, 4, 2 y 1 h). Los cuadrados blancos son ciclos ascendentes en el tiempo (1, 2, 4, 6, 12, 24 y $\mathbf{4 8}$ h) / Average shedding of cercariae in 14 specimens of $P$. purpuratus subjected to 2 experiments with opposite cycles of immersion-emersion. Black squares represent individuals undergoing immersion-emersion cycles descending over time $(48,24,12,6,4,2$ and $1 \mathrm{~h})$. White squares are mussels undergoing immersion-emersion cycles ascending over time (1, 2, 4, 6, 12, 24 and 48 h)

En suma, los resultados muestran que en condiciones de laboratorio: a) no hay diferencias en el consumo de oxígeno entre choritos con y sin parásitos, a una temperatura de $15^{\circ} \mathrm{C}$, b) la liberación de cercarias aumenta con la temperatura del agua, y c) el máximo de la liberación de cercarias se produce en un lapso menor a $2 \mathrm{~h}$ después que el chorito se sumerge.

\section{Discusión}

La ausencia de diferencias significativas en el consumo de oxígeno entre choritos con y sin parásitos sugiere que la demanda energética que le imponen al hospedador no requiere incrementos de su tasa metabólica, aunque en este estudio no se midió si los choritos parasitados incrementan la tasa de consumo de alimento. Esto podría resultar del uso de vías metabólicas anaeróbicas por parte de los parásitos (Tielens 1994, Hechinger 2013). Parte de esta ausencia de diferencias también se puede asociar a la plasticidad fisiológica del hospedador. Por ejemplo, el metabolismo de $P$. purpuratus posee mecanismos de compensación que le permiten conservar la homeostasis durante los ciclos de emersión-inmersión, y que incluyen vías anaeróbicas y aeróbicas (Montecinos et al. 2009).

El hecho de que todos los individuos parasitados presentaran escaso o nulo desarrollo gonadal sugiere que el parásito deriva sus demandas del presupuesto reproductivo del hospedador, lo que coincide con diversos autores que señalan que la presencia de digeneos provoca en el huésped la castración total o parcial (Oliva \& Díaz 1988, Arakelova et al. 2003, Fredensborg et al. 2005, Rice et al. 2006). En otros sistemas Digenea-Gastropoda, se ha observado que la ausencia de diferencias en la tasa metabólica de hospedadores parasitados y no parasitados ocurre sólo cuando la infección es adquirida en una etapa tardía de la ontogenia de los hospedadores, ya que los individuos que habían adquirido la parasitosis en edades tempranas, mostraban mayor consumo de oxígeno que los no parasitados (Arakelova et al. 2003).

El aumento de la liberación de cercarias con la temperatura del agua es un resultado que estaría asociado al incremento del metabolismo del hospedador y del parásito con la temperatura (Poulin 2006, Koprivnikar \& Poulin 2009b). Es decir, aunque el parasitismo no provoque un incremento de la tasa metabólica del hospedador, los aumentos en ésta, producto de la temperatura, podrían favorecer a ciertas poblaciones locales de parásitos a prosperar con el calentamiento global (Koprivnikar \& Poulin 2009a). En condiciones cotidianas, éstas se darían a menores latitudes, en verano, y en sitios más altos del intermareal. Al respecto, se ha registrado que esta parasitosis alcanza mayores prevalencias en el nivel medio-alto del intermareal en Chile central (Muñoz et al. 2013b). 
La liberación de cercarias alcanza valores máximos antes o cerca de $2 \mathrm{~h}$ después de la inmersión del hospedador, y la inmersión prolongada no conduce a mayores valores de ella. Esto indica que el estímulo que induce la liberación de las cercarias es la inmersión. En la naturaleza, los choritos que habitan en el intermareal alto están menos frecuentemente sumergidos y permanecen menos tiempo en dicha condición que los que habitan en la parte inferior del intermareal. Además en dichos sitios se alcanzarían temperaturas más altas, por lo que los choritos de los sectores medio-altos del intermareal serían los que liberarían más cercarias. De hecho, en gasterópodos estuarinos infectados con larvas de digeneos, la liberación de cercarias está estrictamente asociada a niveles altos de marea, es decir, cuando el gasterópodo se encuentra sumergido y cuando hay luz solar (Fingerut et al. 2003, Koprinvnikar \& Poulin 2009a). Otros estudios indican que la liberación de cercarias se asocia a la presencia del siguiente hospedero, aparte de las conveniencias físico-químicas del ambiente al momento de la liberación de estas larvas. Las larvas cercarias tienen un período de infección corto (de 2-8 h), por lo tanto, el parásito de alguna forma asegura el éxito de su transmisión al siguiente hospedador, sincronizando la salida de las cercarias con la presencia de ese hospedador (Lowenberger \& Raw 1994). En consecuencia, la liberación de larvas además de ser influidas por los factores ambientales podría responder a factores biológicos, lo cual produce un escenario complejo al momento de entender la dinámica de liberación larval de digeneos en el momento y lugar en el cual lo hacen. Ciertamente, la interacción entre estos factores requiere ser estudiada en diseños experimentales más complejos.

\section{LITERATURA CITADA}

Abdul-Salam J, BS Sreelatha \& W Al-Kandari. 1997. Temporal variations in the infection of population of Clypcomorus bifasciata (Gastropoda: Prosobranchia) by a digenean microphallid larva in Kuwait Bay. Journal of Helminthology 71: 1-7.

Aldana M, K González, G Loot, J Pulgar \& P Marquet. 2009. First intermediate host of the digenean trematode Proctoeces lintoni (Fellodistomidae) in Chile. The Journal of Parasitology 95: 1408-1414.

Arakelova E, M Chebotareva \& S Zabelinskii. 2003. On adaptive changes of rates of oxygen consumption and lipid metabolism in Littorina saxatilis at parasitic invasions. Journal of Evolutionary Biochemistry and Physiology 39: 519-528.
Broitman B, S Navarrete, F Smith \& S Gaines 2001. Geographic variation of Southeastern Pacific intertidal communities. Marine Ecology Progress Series 224: 21-34.

Cheng T. 1967. Marine molluscs as hosts for symbioses. Advances in Marine Biology 5: 1-424.

Cremonte F, MA Kroeck \& SR Martorelli. 2001. A new monorchiid (Digenea) cercaria parasitising the purple clam Amiantos purpurata (Veneridae) from the Southwest Atlantic Ocean, with notes on its gonadal effect. Folia Parasitologica 48: 217-223.

Cribb T, R Bray \& D Littlewood. 2001. The nature and evolution of the association among digeneans, molluscs and fishes. International Journal for Parasitology 31: 997-1011.

Fingerut J, C Zimmer \& R Zimmer. 2003. Patterns and processes of larval emergence in an estuarine parasite system. The Biological Bulletin 205: 110-120.

Fredensborg B, K Mouritsen \& R Poulin. 2005. Impact of trematodes on host survival and population density in the intertidal gastropod Zeacumantus subcarinatus. Marine Ecology Progress Series 290: 109-117.

Fried B, R Laterra \& Y Kim. 2002. Emergence of cercariae of Echinostoma caproni and Schistosoma mansoni from Biomphalaria glabrata under different laboratory conditions. Journal of Helminthology 76: 369-371.

Hechinger R. 2013. A metabolic and body-size scaling framework for parasite within-host abundance, biomass, and energy flux. The American Naturalist 182: 234-248.

Koprivnikar J \& R Poulin. 2009a. Effects of temperature, salinity, and water level on the emergence of marine cercariae. Parasitology Research 105: 957-965.

Koprivnikar J \& R Poulin. 2009b. Interespecific and intraespecific variation in cercariae release. The Journal of Parasitology 95: 14-19.

Lo C \& K Lee. 1996. Pattern of emergence and the effects of temperature and light on the emergence and survival of heterophyid cercariae (Centrocestus formosanus and Haplorchis pumilio). The Journal of Parasitology 82: 347350.

Lowenberger CA \& ME Rau. 1994. Plagiorchis elegans: emergence, longevity and infectivity of cercariae, and host behavioural modifications during cercarial emergence. Parasitology 109: 65-72.

Lyholt H \& K Buchmann. 1996. Diplostomum spathaceum: Effects of temperature and light on cercarial shedding and infection of rainbow trout. Diseases of Aquatic Organisms 25: 169-173.

Marcogliese D. 2001. Implications of climate change for parasitism of animals in the aquatic environment. Canadian Journal of Zoology 79: 1331-1352.

Marcogliese D. 2008. The impact of climate change on the parasites and infectious diseases of aquatic animals. Revue Scientifique et Technique (International Office of Epizootics) 27: 467-484. 
Meyrowitsch D, N Christensen \& O Hindsbo. 1991. Effects of temperature and host density on the snail-finding capacity of cercariae of Echinostoma-caproni (Digenea, Echinostomatidae). Parasitology 102: 391-395.

Montecinos L, J Cisterna, C Cáceres \& G Saldías. 2009. Equilibrio ácido-base durante la exposición aérea en el molusco bivalvo Perumytilus purpuratus (Lamarck, 1819) (Bivalvia: Mytilidae). Revista de Biología Marina y Oceanografía 44: 181-187.

Morley N. 2011. Thermodynamics of cercarial survival and metabolism in a changing climate. Parasitology 138: 14421452 .

Mouritsen K. 2002. The Hydrobia ulvae-Maritrema subdolum association: Influence of temperature, salinity, light, water-pressure and secondary host exudates on cercarial emergence and longevity. Journal of Helminthology 76: 341-347.

Muñoz G, Z López \& L Cárdenas. 2013a. Morphological and molecular analyses of larval trematodes in the intertidal bivalve Perumytilus purpuratus of Central Chile. Journal of Helminthology 87(3): 356-363.

Muñoz G, P Torres, J Valdés \& A Rodríguez. 2013b. Spatiotemporal variation in the prevalence of trematodes in the bivalve Perumytilus purpuratus. Acta Parasitologica 58: 155-166.

Noble E \& G Noble. 1965. Parasitología: Biología de los parásitos animales, $696 \mathrm{pp}$. Interamericana, México.

Oliva ME \& M Díaz. 1988. Aspectos cuantitativos de la infección por Proctoeces humboldti (Trematoda: Fellodistomidae) en la lapa Fissurella crassa (Mollusca: Archaeogastropoda). Revista Chilena de Historia Natural 61: 27-33.
Oliva M, I Valdivia, L Cárdenas, M George-Nascimento, K González, R Guiñez \& D Cuello. 2010. Molecular and experimental evidence refuse the life cycle of Proctoeces lintoni (Fellodistomidae) in Chile. Parasitology Research 106: 737-740.

Olmos V \& M George-Nascimento. 1997. El gremio de las larvas de Digenea en el caracol del sur de Chile Chilina dombeyana: ¿Qué indica la tasa metabólica de los hospedadores parasitados? Revista Chilena de Historia Natural 70: 109-118.

Poulin R. 2006. Global warming and temperature-mediated increases in cercarial emergence in trematode parasites. Parasitology 132: 143-151.

Rice T, E Mcgraw, E O’Brien, A Reverter, D Jackson \& B Degnan. 2006. Parasitic castration by the digenean trematode Allopodocotyle sp. alters gene expression in the brain of the host mollusk Haliotis asinina. Federation of European Biochemical Societies Letters 580: 3769-3774.

Shim K, J Koprivnikar \& M Forbes. 2013. Variable effects of increased temperature on a trematode parasite and its intertidal host. Journal of Experimental Marine Biology and Ecology 439: 61-68.

Studer A, D Thieltges \& R Poulin. 2010. Parasites and global warming: net effects of temperature on an intertidal hostparasite system. Marine Ecology Progress Series 415: 1122.

Tielens A. 1994. Energy generation in parasitic helminths. Parasitology Today 10: 346-352.

Zar J. 1999. Biostatistical analysis, 718 pp. Prentice Hall, Upper Saddle River. 